Bundesgesundheitsbl 2015 · 58:762-768

DOI 10.1007/s00103-015-2173-y

Online publiziert: 28. Mai 2015

c) Springer-Verlag Berlin Heidelberg 2015

Bekanntmachung des Umweltbundesamtes

\title{
Gesundheitliche Bewertung von Trichlorethen in der Innenraumluft
}

\author{
Mitteilung der Ad-hoc-Arbeitsgruppe \\ Innenraumrichtwerte der Kommission \\ Innenraumlufthygiene und der Obersten \\ Landesgesundheitsbehörden
}

\section{Stoffidentifizierung [3]}

\begin{tabular}{|ll}
\hline Systematischer Name: & Trichlorethen \\
\hline Synonyme: & $\begin{array}{l}\text { Trichlorethylen, } \\
\text { 1,1,2-Trichlorethen, } \\
\text { Ethylentrichlorid, } \\
\text { TRI, TCE }\end{array}$ \\
\hline CLP-Index-Nr.: & $602-027-00-9$ \\
\hline EG-Nr:: & $201-167-4$ \\
\hline CAS-Nr.: & $79-01-6$ \\
\hline Summenformel: & $\mathrm{C}_{2} \mathrm{HCl}_{3}$ \\
\hline Strukturformel: & \\
&
\end{tabular}

\subsection{Physikalisch-chemische} Eigenschaften $[3,4]$

Trichlorethen ist eine farblose, nichtbrennbare Flüssigkeit mit einem süßlichetherischen, an Trichlormethan („Chloroform“) erinnernden Geruch [5].

\begin{tabular}{|ll}
\hline Molekulargewicht: & $131,39 \mathrm{~g} / \mathrm{mol}$ \\
\hline Schmelzpunkt: & $-84,8^{\circ} \mathrm{C}$ \\
\hline Siedepunkt: & $86,9^{\circ} \mathrm{C}$ (bei $\left.1013 \mathrm{hPa}\right)$ \\
\hline Dichte: & $1,465 \mathrm{~g} / \mathrm{cm}^{3}$ (bei $\left.20^{\circ} \mathrm{C}\right)$ \\
\hline Dampfdruck: & $86 \mathrm{hPa}$ (bei $\left.20^{\circ} \mathrm{C}\right)$ \\
\hline Wasserlöslichkeit: & $1,1 \mathrm{~g} / \mathrm{l}\left(\right.$ bei $\left.20^{\circ} \mathrm{C}\right)$ \\
\hline log Poctanol/Wasser: & 2,29 \\
\hline Umrechnung & $1 \mathrm{ml} / \mathrm{m}^{3}=5,47 \mathrm{mg} / \mathrm{m}^{3} ;$ \\
$\left(20^{\circ} \mathrm{C}, 1013 \mathrm{hPa}\right):$ & $1 \mathrm{mg} / \mathrm{m}^{3}=0,183 \mathrm{ml} /$ \\
& $\mathrm{m}^{3}[5]$ \\
\hline
\end{tabular}

\section{Exposition}

Infolge des Verbots der verbrauchernahen Verwendung von Trichlorethen, z. B. in Chemischreinigungsanlagen [6] Anfang der neunziger Jahre lässt sich Trichlorethen außerhalb industrieller Arbeitsplätze kaum noch in der Innenraumluft von Gebäuden in Deutschland nachweisen. Beispielsweise lag im Kinderumweltsurvey von 2003-2006, in dem die Innenraumluft überwiegend von Kinderzimmern mithilfe von Passivsammlern über den Zeitraum von einer Woche erfasst wurde, das 95. Perzentil unterhalb der Bestimmungsgrenze von $1 \mu \mathrm{g}$ Trichlorethen $/ \mathrm{m}^{3}$, der Maximalwert betrug $64 \mu \mathrm{g}$ Trichlorethen $/ \mathrm{m}^{3}$ [7]. Untersuchungen in verschiedenen Innenräumen (z. B. Wohnungen, Schulen, öffentliche Gebäude, Büroräume) zwischen 2002 und 2006 ergaben bei einer Bestimmungsgrenze von $1 \mu \mathrm{g}$ Trichlorethen $/ \mathrm{m}^{3}$ ein 95 . Perzentil von $1,2 \mu \mathrm{g} / \mathrm{m}^{3}$ und einen Maximalwert von $125 \mu \mathrm{g} / \mathrm{m}^{3}$ [8]. Erhöhte TrichlorethenKonzentrationen in der Innenraumluft stammen häufig aus Einträgen von Altlasten im oder am Gebäude.

\section{Toxikokinetik}

Eingeatmetes Trichlorethen wird nur in geringem Maße (etwa $10 \%$ [9]) wieder unverändert abgeatmet. Überwiegend diffundiert es als lipofile Substanz schnell durch die Membranen der Lun- 
genbläschen. Es verteilt sich im Körper, passiert die Blut-Hirn- und die Plazentaschranke und reichert sich in fettreichen Geweben an [10]. Der Anteil des aus der Gasphase über die Haut aufgenommenen Trichlorethens ist mit 0,3\% gering [11].

Aufgenommenes Trichlorethen wird im Wesentlichen in der Leber oxidiert, Teilschritte einer reduktiven Verstoffwechselung finden auch in der Niere statt, allerdings in sehr geringem Ausmaß. Untersuchungen an Probanden und an Beschäftigten, die gegenüber Trichlorethen exponiert waren, sowie auch an der Ratte ergaben, dass die Menge an Metaboliten aus dem reduktiven Abbau um mehr als drei Größenordnungen geringer ist als diejenige aus dem oxidativen Abbau [12-14]. Im Vergleich mit den oxidierten Metaboliten weisen die reduktiven Stoffwechselprodukte jedoch eine größere Instabilität und damit eine höhere Toxizität, insbesondere in der Niere, auf (s. u.).

Die durch CYP2E1 und weitere Cytochrom P450-Enzyme vermittelte Oxidation von Trichlorethen führt beim Menschen über ein hypothetisches Trichlorethenepoxid zum Trichlorethanal (älterer Name „Chloral“) bzw. 2,2,2-Trichlorethandiol („Chloralhydrat") und weiter zu den Hauptmetaboliten 2,2,2-Trichlorethanol (TCOH) und dessen Glucuronid (TCOG) sowie in geringerem Maße zur Trichlorethansäure („Trichloressigsäure" - TCA). Weitere Endprodukte dieses Abbauweges sind Dichlorethansäure und Monochlorethansäure sowie N-Hydroxyacetylaminoethanol (vermutet wird eine Reaktion des Epoxids mit Phosphatidylaminoethanol) [15]. Im Urin von exponierten Labortieren ließen sich weitere Metabolite wie z. B. Ethandisäure („Oxalsäure“) und Ethanalsäure („Glyoxylsäure") nachweisen.

Der reduktive Stoffwechselweg ist vor allem bei der Sättigung der Oxidation durch eine hohe Exposition gegenüber Trichlorethen bedeutsam. Durch Konjugation mit Glutathion mithilfe der Glutathion-S-transferase in der Leber entstehen hierbei zunächst S-(1,2-Dichlorvinyl)glutathion (DCVG) und in geringerem Maße sein Isomer S-(2,2-Dichlorvinyl)glutathion [16]. Die Bildung von DCVG ist bei Männern teilweise höher als bei Frauen; dies weist auf einen Polymorphismus der Glutathion-Konjugation hin [17]. S(1,2-Dichlorvinyl)glutathion (der weitere Abbau für sein Isomer verläuft entsprechend und wird hier nicht dargestellt) wird durch die in Epithelzellen der proximalen Tubuli der Niere vorkommenden hydrolytischen Enzyme $\gamma$-Glutamyltransferase und Cysteinylglycindipeptidase in das S-(1,2-Dichlorvinyl)-L-cystein (DCVC) umgewandelt. DCVC kann entweder mithilfe der N-Acetyltransferase zum N-Acetyl-S-(1,2-dichlorvinyl)-L-cystein (NAcDCVC) acetyliert, durch eine Cystein-Konjugat- $\beta$-Lyase in das reaktive S-(1,2-Dichlorovinyl)thiol (DCVSH) umgewandelt oder durch eine Monooxygenase in eine weitere reaktive Verbindung, das S-(1,2-Dichlorvinyl)-L-cysteinsulfoxid (DCVCSO), oxidiert werden. Das durch die $ß$-Lyase gebildete instabile DCVSH wandelt sich spontan in ein Chlorthioketen oder in ein Chlorthionacetylchlorid um. Beide Verbindungen sind hochreaktiv und können kovalente Addukte mit Nukleinsäuren oder anderen Proteinen bilden [10].

Von den Reaktionsprodukten des DCVC konnte bisher nur das NAcDCVC sowohl im Urin von Ratten und Mäusen $[17,18]$ als auch beim Menschen identifiziert werden [13, 14, 17].

Aus den Untersuchungen an Mensch, Ratte und Maus lassen sich keine qualitativen, wohl aber quantitative Unterschiede im Stoffwechsel erkennen. Im Vergleich zum Menschen weisen vor allem männliche Mäuse eine höhere metabolische Kapazität auf, so dass bei diesen Tieren besonders hohe TCA-Konzentrationen im Blut mit entsprechenden kritischen Konzentrationen in einzelnen Organen wie z. B. der Leber auftraten [15]. In Leberzellen von Mäusen und Ratten fanden sich etwa 7 bis 125-mal mehr DCVG als in menschlichen Leberzellen [19].

Die Ausscheidung von Trichlorethen beim Menschen lässt sich durch ein 3-Kompartment-Modell mit Halbwertzeiten von 2-3 min für gut durchblutete, von einer halben Stunde für weniger durchblutete und von 3,5-5 h für fettreiche Gewebe beschreiben [20]. Die Eliminationshalbwertzeit von Trichlorethanol aus dem Blut beträgt etwa $10 \mathrm{~h}$ (Bandbreite 7-12 h), von Trichlorethansäure wegen seiner Bindung an Eiweiße im Plasma 2 Tage (1,6-
3 Tage) [9]. In Nagetieren sind die Ausscheidungshalbwertzeiten aus dem Plasma für die Metaboliten von Tetrachlorethen deutlich kürzer. Die Ausscheidung der Stoffwechselprodukte von Trichlorethen erfolgt im Wesentlichen über den Harn. Trichlorethansäure-Konzentrationen im Urin von Männern sind etwa doppelt so hoch im Vergleich mit Frauen, bei jüngeren Menschen höher als bei älteren [21].

Eine Alkoholzufuhr oder eine Exposition gegenüber anderen halogenierten Kohlenwasserstoffen kann den Abbau von Trichlorethen in Abhängigkeit von den Expositionsbedingungen beeinträchtigen [22, 23] oder anregen [24].

\section{Gesundheitliche Wirkungen}

Eingeatmetes Trichlorethen weist vor allem neurotoxische, nephrotoxische, mutagene und krebserzeugende Wirkungen auf. Für andere Endpunkte (z. B. Immuntoxizität) liegen einzelne Hinweise vor oder die Wirkungen traten erst bei hohen Dosen auf. Die meisten Gremien sehen vor allem die nierenschädigende, mutagene und krebserzeugende Wirkung von Trichlorethen beim Menschen als kritische Endpunkte an (z. B. [3, 5]). Im Folgenden werden vor allem Wirkungen im niedrigen Expositionsbereich dargestellt; für eine Gesamtdarstellung der gesundheitlichen Wirkungen von Trichlorethen sei auf verschiedene Übersichten verwiesen $[3-5,10,25]$.

\subsection{Neurotoxizität}

In einer älteren Arbeitsplatzstudie zeigten Beschäftigte Müdigkeit und erste motorische Koordinationsstörungen ab einer durchschnittlichen Konzentration von $88 \mathrm{mg}$ Trichlorethen $/ \mathrm{m}^{3}$ [26]. Zur neurotoxischen Wirkung von Trichlorethen liegen weitere Studien bei meist höheren Expositionskonzentrationen vor; allerdings sind die Expositionsbedingungen in diesen Studien häufig nicht hinreichend beschrieben [5].

Murata et al. [27] beobachteten Gleichgewichtsstörungen und Tremor der Hände bei 57 Arbeitnehmern, die im Mittel 11 Jahre gegenüber Trichlorethen exponiert waren, im Vergleich zu einer Kontroll- 
gruppe $(n=60)$. Auch in dieser Studie waren die vorangegangenen Expositionsbedingungen nicht hinreichend bekannt.

\subsection{Nephrotoxizität}

Nach einer Exposition gegenüber durchschnittlich $175 \mathrm{mg}$ Trichlorethen $/ \mathrm{m}^{3}$ (3$1400 \mathrm{mg} / \mathrm{m}^{3}$ ) über im Mittel 4 Jahre (1-20 Jahre) wiesen 70 Beschäftige im Vergleich zur Kontrollgruppe signifikant erhöhte Konzentrationen von Albumin und NAcetyl-ß-D-glucosaminidase (NAG) als erste präklinische Anzeichen einer nierenschädigenden Wirkung auf [28]. Nach Exposition gegenüber 33-55 mg Trichlorethen $/ \mathrm{m}^{3}$ zeigten sich bei den Beschäftigten keine nephrotoxischen Effekte [29].

In einer neuen Studie wurden frühe Nierenschädigungen durch Trichlorethen anhand empfindlicher Biomarker wie KIM-1-und pi-GST untersucht [30]. Das menschliche Membranprotein Kidney injury molecule-1 (KIM-1) zeigt vor allem Schädigungen in den proximalen Nierentubuli an. Glutathion-S-transferase $\pi$ (pi-GST) stammt überwiegend aus den distalen Tubuli. Bei Beschäftigen ( $n=80$, Kontrollgruppe $n=45$ ) nahm bereits nach einer kurzen Expositionsdauer von durchschnittlich 2 Jahren bei eher niedrigen Trichlorethen-Konzentrationen am Arbeitsplatz (Median 66 mg/m³) KIM-1 und - weniger ausgeprägt - pi-GST dosisabhängig zu. Sowohl für die aktuelle als auch für die kumulative Exposition zeigte sich für KIM-1 ein hoch signifikanter Zusammenhang in der linearen Regression $(p=0,0001)$. Für pi-GST war die Signifikanz geringer ( $p=0,06$ bzw. 0,05$)$. Für die Expositionsgruppe $>66 \mathrm{mg} / \mathrm{m}^{3}$ betrug $p=0,0008$ im Vergleich zur Kontrollgruppe, selbst für die Expositionsgruppe $<66 \mathrm{mg}$ Trichlorethen $/ \mathrm{m}^{3}$ ergab sich mit $p=0,02$ noch eine Signifikanz. Eine weitere Differenzierung dieser Expositionsgruppe wurde jedoch nicht vorgenommen. Erhöhte Werte der oben genannten Biomarker für ausgeprägte Nierenschäden wie z. B. NAG wurden nicht beobachtet.

\subsection{Kanzerogenität}

In einer Vielzahl von Arbeitsplatzstudien wurde ein vermuteter Zusammen-

Bundesgesundheitsbl 2015 · 58:762-768 DOI 10.1007/s00103-015-2173-y

๑) Springer-Verlag Berlin Heidelberg 2015

Bekanntmachung des Umweltbundesamtes

Gesundheitliche Bewertung von Trichlorethen in der
Innenraumluft. Mitteilung der Ad-hoc-Arbeitsgruppe
Innenraumrichtwerte der Kommission Innenraumluft-
hygiene und der Obersten Landesgesundheitsbehörden

\section{Zusammenfassung}

Trichlorethen ist im europäischen Gefahrstoffrecht als wahrscheinlich krebserzeugend beim Menschen und als möglicherweise mutagen eingestuft. Nach Auffassung mehrerer Ausschüsse (Ausschuss für Gefahrstoffe, europäischer Wissenschaftlicher Ausschuss für Expositionsbegrenzungen am Arbeitsplatz, Risikobewertungsausschuss der Europäischen Chemikalienbehörde (ECHA-RAC)) können Trichlorethen-Konzentrationen, die in den Tubuli der Niere zytotoxisch wirken, das Risiko der Entstehung von Nierenkrebs erhöhen. Bei nicht zytotoxischen Trichlorethen-Konzentrationen ist ein viel geringeres Krebsrisiko anzunehmen. Zur Abschätzung des Krebsrisikos der Allgemeinbevölkerung durch eingeatmetes Trichlorethen hat der ECHA-RAC deshalb eine sublineare Expositions-Wirkungsbeziehung der krebserzeugenden Wirkung von Trichlorethen angenommen und ein theoreti- sches Risiko, nach lebenslanger Exposition gegenüber Trichlorethen an Krebs zu erkranken, von $6,4 \times 10^{-5}\left(\mathrm{mg} / \mathrm{m}^{3}\right)^{-1}$ unterhalb der NOAEC für renale Zytotoxizität von $6 \mathrm{mg}$ Trichlorethen $/ \mathrm{m}^{3}$ abgeschätzt. Hieraus ergibt sich ein theoretisches Lebenszeitrisiko von $10^{-6}$ bei einer Konzentration von $0,02 \mathrm{mg}$ Trichlorethen $/ \mathrm{m}^{3}$. Diese Konzentration liegt oberhalb des Referenzwertes von Trichlorethen in der Innenraumluft. Die Ad-hoc-Arbeitsgruppe Innenraumrichtwerte empfiehlt auf dieser Grundlage einen risikobasierten Leitwert von 0,02 mg Trichlorethen $/ \mathrm{m}^{3} \mathrm{In}$ nenraumluft. Sie betrachtet expositionsmindernde Maßnahmen unterhalb des Leitwertes als nicht angemessen.

\section{Schlüsselwörter}

Trichlorethen · Innenraumluft · Zytotoxizität . Nierenkrebs · Risikobewertung · Leitwert

\section{Health evaluation of trichloroethylene in indoor air. Communication from the German ad-hoc working group on indoor guidelines of the Indoor Air Hygiene Committee and of the states' supreme health authorities}

\begin{abstract}
In the European Hazardous Substances Regulation No 1272/2008 trichloroethylene has been classified as a probable human carcinogen and a suspected mutagen. According to several Committees (German Committee on Hazardous Substances, European Scientific Committee on Occupational Exposure Limits, European Chemicals Agency's Committee for Risk Assessment (ECHA-RAC)) concentrations of trichloroethylene cytotoxic to renal tubuli may increase the risk to develop renal cancer. At non-cytotoxic concentrations of trichloroethylene a much lower cancer risk may be assumed. Therefore, evaluating the cancer risk to the public following inhalation of trichloroethylene ECHA-RAC has assumed a sublinear exposure-response relationship for carcinogenicity of trichloroethylene. Specifically,
\end{abstract}

ECHA-RAC assessed a cancer risk of $6.4 \times 10^{-5}$ $\left(\mathrm{mg} / \mathrm{m}^{3}\right)^{-1}$ following life time exposure to trichloroethylene below a NOAEC for renal cytotoxicity of $6 \mathrm{mg}$ trichloroethylene $/ \mathrm{m}^{3}$. Further evaluation yields a life-time risk of $10^{-6}$ corresponding to $0.02 \mathrm{mg}$ trichloroethylene $/ \mathrm{m}^{3}$. This concentration is well above the reference (e.g. background) concentration of trichloroethylene in indoor air. Consequently the Adhoc Working Group on Indoor Guidelines recommends $0.02 \mathrm{mg}$ trichloroethylene $/ \mathrm{m}^{3}$ as a risk-related guideline for indoor air. Measures to reduce exposure are considered inappropriate at concentrations below this guideline.

\section{Keywords}

trichloroethylene · indoor air · cytotoxicity . renal cancer · risk evaluation · guideline hang einer Trichlorethen-Exposition von Beschäftigten mit Nierenzellkarzinomen, Lebertumoren, Non-Hodgkin-Lymphomen (NHL) sowie weiteren Tumoren untersucht. Die Ergebnisse der einzelnen
Studien waren widersprüchlich; nur in wenigen Studien fand sich eine sehr hohe Exposition gegenüber Trichlorethen, vielfach lag auch eine Exposition gegenüber weiteren chlorierten Lösungsmitteln 
vor und der Einfluss des Tabakrauchens, einer bekannten Ursache für Nierenkrebs, konnte nicht immer sicher ausgeschlossen werden [4].

\subsubsection{Nierenkrebs}

In einer Metaanalyse von 24 Kohortenund Fall-Kontroll-Studien und 3 Studien nach hoher Exposition gegenüber Trichlorethen ergab sich ein relatives Risiko in der Metaanalyse (meta-RR) von 1,27 insgesamt (95\%-Konfidenzintervall: 1,131,43) und für die höchste Expositionsgruppe ein meta-RR von 1,58 (95\%-Konfidenzintervall: 1,28-1,96) [31]. Eine Metaanalyse des US-amerikanischen Nationalen Krebsinstituts von 28 Kohorten- und Fall-Kontroll-Studien wies ebenfalls einen Zusammenhang zwischen beruflicher Trichlorethen-Exposition und Nierenkrebs nach [32].

Nierenzellkarzinome traten vor allem bei sehr hohen Trichlorethen-Konzentrationen, z. B. oberhalb des früheren MAKWertes von $275 \mathrm{mg} / \mathrm{m}^{3}$ auf, bei denen auch bereits nicht-neoplastische Nierenschädigungen beobachtet worden waren $[33,34]$. Ein erhöhtes Risiko für Nierenkrebs durch Trichlorethen zeigte sich bei Beschäftigten mit einem aktiven Glutathion-S-Transferase (GSTT1)-Gentyp [35].

\subsubsection{Non-Hodgkin-Lymphom (NHL)}

Ein möglicher Zusammenhang zwischen einer beruflichen Exposition gegenüber Trichlorethen und NHL wurde anhand von 8 Kohorten- und 8 Fall-KontrollStudien geprüft. In der Metaanalyse ergab sich ein meta-RR von 1,2 (95\%-KI: $1,1-1,4)$ insgesamt und von 1,4 (95\%-KI: 1,1-1,8) für die höchste Expositionsstufe. Insgesamt wurde ein Zusammenhang mit NHL als weniger belastbar angesehen [31]. In einer weiteren Auswertung zeigte sich weder hinsichtlich der Höhe noch hinsichtlich der Dauer der Exposition gegenüber Trichlorethen ein Zusammenhang mit NHL [36].

\subsubsection{Leberkrebs}

Anhand von 9 Kohortenstudien wurde der Zusammenhang mit Leberkrebs als eingeschränkt belastbar eingestuft [31].

\subsubsection{Tierexperimentell}

Bei verschieden Rattenstämmen und bei beiden Geschlechtern wurden nach Inhalation oder oraler Gabe von Trichlorethen geringe Anstiege von Nierentumoren beobachtet, signifikant war die Inzidenz nur bei männlichen F344/N-Ratten [37]. Dabei ist von Bedeutung, dass Nierentumore in keiner der Kontrollgruppen auftraten. Bei männlichen Tieren einzelner Rattenstämme fanden sich auch eine Leukämie sowie Leydig-Zelltumore im Hoden [38, 40].

Nach Inhalation oder oraler Gabe von Trichlorethen traten bei B6C3F1-Mäusen beiderlei Geschlechts und bei männlichen Swiss-Mäusen vermehrt Leber- und Lungentumore sowie bei weiblichen Tieren Lymphome auf [38, 39, 41].

\subsection{Mutagenität}

In in-vitro- und in tierexperimentellen in-vivo-Untersuchungen erwies sich Trichlorethen selbst überwiegend als schwach oder nicht mutagen. In-vivoDaten zur Mutagenität von Trichlorethen beim Menschen waren widersprüchlich [42]. Eine mutagene Aktivität konnte hingegen für mehrere Metaboliten des Trichlorethens gezeigt werden [10]. Trichlorethanal bzw. sein Hydrat Trichlorethandiol waren sowohl in-vitro als auch in-vivo gentoxisch. Für eine Mutagenität von Dichlorethansäure liegen schwache Hinweise vor. Zur Mutagenität von Trichlorethanol liegen nur sehr wenige, widersprüchliche in-vitro-Daten vor.

Von den Produkten des reduktiven Stoffwechsels erwiesen sich DCVG und vor allem DCVC als direktwirkende $\mathrm{Mu}$ tagene in-vitro und in-vivo. DCVCSO und NAcDCVC waren in-vitro ebenfalls gentoxisch $[10,51]$.

\subsection{Geruchswahrnehmung}

In einer älteren Studie wurde als niedrigste Konzentration, ab der Trichlorethen geruchlich wahrgenommen wurde, $46 \mathrm{mg} /$ $\mathrm{m}^{3}$ genannt [44]. In einer als belastbar angesehenen neueren Untersuchung ergab sich eine Geruchswahrnehmungsschwelle von 5,3 mg Trichlorethen $/ \mathrm{m}^{3}$ [45].

\section{Bewertung}

Sowohl beim oxidativen als vor allem auch beim reduktiven Abbau von Trichlorethen bilden sich mutagene Metabolite. Angesichts der Bildung der gentoxischen Verbindungen DCVG und vor allem DCVC in der Niere und dem Nachweis von Nierenkrebs beim Menschen nach sehr hohen Expositionen gegenüber Trichlorethen wurden in den neueren Bewertungen überwiegend epidemiologische Studien an Arbeitsplätzen für die Aufstellung von Dosis-Wirkungsbeziehungen herangezogen. Nierentumore traten auch bei Ratten nach chronischer Inhalation von Trichlorethen auf, jedoch nicht bei Mäusen.

Beim derzeitigen Kenntnisstand ist es fraglich, ob eine Exposition gegenüber Trichlorethen auch zu Leberkrebs oder Non-Hodgkin-Lymphomen beim Menschen führen kann. Nach Auffassung des Ausschusses für Gefahrstoffe lassen Dosis-Wirkungsabschätzungen für diese Tumoren im Vergleich zur Auslösung von Nierentumoren ein höheres Krebsrisiko für andere Organe wenig wahrscheinlich erscheinen. Für Lebertumore und NonHodgkin-Lymphome fehlen auch belastbare Hinweise auf einen gentoxischen Mechanismus durch Trichlorethen oder seine Stoffwechselprodukte [46].

Die Nierenschädigung stellt auch den empfindlichsten Endpunkt der nicht-kanzerogenen Wirkung von Trichlorethen dar. In den Arbeitsplatzstudien fand sich ein erhöhtes Nierenkrebsrisiko bei hohen Trichlorethen-Konzentrationen mit sehr hohen Expositionsspitzen zusammen mit tubulären Nierenschäden. Es wird angenommen, dass zytotoxische Effekte in den Tubuli der Niere die Entstehung von Nierenkrebs verstärken und deshalb das Nierenkrebsrisiko bei Trichlorethen-Konzentrationen ohne zytotoxische Wirkung sehr viel geringer ist. Nach Ansicht des Ausschusses für Gefahrstoffe [46], des europäischen Wissenschaftlichen Ausschusses für Expositionsbegrenzungen am Arbeitsplatz [5] und des Risikobewertungsausschusses der Europäischen Chemikalienbehörde [3] erscheint deshalb eine sublineare Dosis-Wirkungsbeziehung plausibel, d. h. das Krebsrisiko sollte erst oberhalb von nierenschädigenden Konzent- 


\begin{tabular}{|c|c|c|c|c|}
\hline Gremium & Wirkungsmechanismus & $\begin{array}{l}\text { Extrapola- } \\
\text { tion }\end{array}$ & $\begin{array}{l}\text { Kritische Stu- } \\
\text { die/Effekt }\end{array}$ & $\begin{array}{l}\text { Risiko für die All- } \\
\text { gemein-bevölkerung } \\
\left(\mathrm{mg} / \mathrm{m}^{3}\right)^{-1}\end{array}$ \\
\hline$[47,48]$ & Gentoxizität & $\begin{array}{l}\text { Linear ohne } \\
\text { Schwelle }\end{array}$ & $\begin{array}{l}\text { [38] Ratte, } \\
\text { inhalativ: Leydig- } \\
\text { Tumore }\end{array}$ & $4,3 \times 10^{-4}$ \\
\hline [49] & Gentoxizität & $\begin{array}{l}\text { Linear ohne } \\
\text { Schwelle }\end{array}$ & $\begin{array}{l}\text { [50] Arbeitsplatz: } \\
\text { Nierenkrebs }\end{array}$ & $4 \times 10^{-3}$ \\
\hline [3] & Gentoxizität/Zytotoxizität & $\begin{array}{l}\text { Sublinear oh- } \\
\text { ne Schwelle }\end{array}$ & $\begin{array}{l}{[28,29,33,51,} \\
52] \\
\text { Arbeitsplatz: Nie- } \\
\text { renkrebs }\end{array}$ & $\begin{array}{l}6,4 \times 10^{-5} \\
\text { (unterhalb von } 6 \mathrm{mg} / \\
\mathrm{m}^{3} \text { ) }\end{array}$ \\
\hline
\end{tabular}

rationen von Trichlorethen nennenswert steigen.

\subsection{Einstufungen/Bewertungen}

Trichlorethen ist in der EU als krebserzeugend in der Kategorie Canc. 1 B („wahrscheinlich beim Menschen krebserzeugend") und als mutagen in der Kategorie 2 („möglicherweise mutagen“) eingestuft [1]. Die Internationale Krebsforschungsbehörde (IARC) hat Trichlorethen kürzlich als krebserzeugend beim Menschen bezeichnet [4]. Nach Ansicht der IARC liegen ausreichende Hinweise für einen Zusammenhang einer Exposition gegenüber Trichlorethen und Nierenkrebs sowie eingeschränkte Hinweise für NonHodgkin-Lymphome und Leberkrebs vor.

Der europäische Wissenschaftliche Ausschuss für Expositionsbegrenzungen am Arbeitsplatz entnahm aus der Arbeitsplatzstudie von Green et al. [28] eine niedrigste beobachtete nachteilige Wirkungskonzentration von $175 \mathrm{mg}$ Trichlorethen/ $\mathrm{m}^{3}$ für den Endpunkt Nephrotoxizität und leitete einen Arbeitsplatzgrenzwert von $55 \mathrm{mg}$ Trichlorethen $/ \mathrm{m}^{3}\left(10 \mathrm{ml} / \mathrm{m}^{3}\right)$ ab [5] .

Der Ausschuss für Gefahrstoffe schätzte eine Trichlorethen-Konzentration am Arbeitsplatz von $60 \mathrm{mg} / \mathrm{m}^{3}$ für ein Toleranzrisiko von 4:1000 und von $33 \mathrm{mg} /$ $\mathrm{m}^{3}$ für ein Akzeptanzrisiko von übergangsweise 4:10.000 ab. Es ist angedacht, dieses übergangsweise Akzeptanzrisiko spätestens ab 2018 auf 4:100.000 zu senken, dies entspräche einer Arbeitsplatzkonzentration von $3 \mathrm{mg}$ Trichlorethen/ $\mathrm{m}^{3}$ [46]. Nach Auffassung des Ausschusses ist unterhalb einer Konzentration von $33 \mathrm{mg}$ Trichlorethen $/ \mathrm{m}^{3}$ am Arbeitsplatz nicht mehr mit einer Nierenschädigung zu rechnen (NOAEC), auf dessen Boden sich im Sinne einer Promotion/Progression ein klinisch manifester Tumor entwickeln könnte. Für Konzentrationen unterhalb von $33 \mathrm{mg}$ Trichlorethen $/ \mathrm{m}^{3}$ ging der Ausschuss deshalb von einer grundsätzlich zu erwartenden Sublinearität aus und nahm am NOAEC von $33 \mathrm{mg}$ Trichlorethen $/ \mathrm{m}^{3}$ im Sinne einer Konvention eine Abnahme des Krebserkrankungsrisikos um zwei Größenordnungen bei Abnahme der Exposition um eine Größenordnung an.

Hinsichtlich des Krebsrisikos für die Allgemeinbevölkerung durch eingeatmetes Trichlorethen liegen drei Bewertungen vor (• Tab. 1):

Die Weltgesundheitsorganisation [48] hatte 2010 bei ihrer Ableitung von Leitwerten für die Innenraumluftqualität keine neue Risikoabschätzung durchgeführt, sondern eine frühere Bewertung [47] übernommen. Sie verwendete dabei eine chronische Inhalationstudie an Ratten, in der Leydig-Zelltumoren beobachtet wurden [38]. Die Wahl dieses Endpunktes und die auf der Grundlage dieser Studie durchgeführte Risikoabschätzung erscheinen angesichts der neueren epidemiologischen Studien nicht mehr zeitgemäß.

Die US-amerikanische Umweltschutzbehörde [42] zog die relativ kleine Arbeitsplatzstudie von Charbotel et al. [50] ( $n=86$, Kontrollgruppe $n=316$ ) heran. Da die vorangegangene Exposition in dieser Studie nur semiquantitativ (auf der Basis von Telefoninterviews und durch Transformation von Jobbeschreibungen in Expositionskategorien) ermittelt wur- de, ist diese Risikoabschätzung mit erheblichen Unsicherheiten behaftet.

In Rahmen der Zulassung von Trichlorethen in industriellen Anwendungen hat der Risikobewertungsausschuss der Europäischen Chemikalienbehörde auch mögliche gesundheitliche Auswirkungen auf die Allgemeinbevölkerung abgeschätzt. In Anlehnung an das vom Ausschuss für Gefahrstoffe vorgeschlagene Verfahren [46] geht der Risikobewertungsausschuss zur Ableitung einer sogenannten Referenz-Dosis-Wirkungsbeziehung der krebserzeugenden Wirkung ebenfalls von einem sublinearen Verlauf des Krebsrisikos durch Trichlorethen aus und leitet zum Schutz der Allgemeinbevölkerung nach lebenslanger Exposition (Annahme: 70 Jahre) ein zusätzliches Krebsrisiko von $6,4 \times 10^{-5}$ (mg Trichlorethen $\left./ \mathrm{m}^{3}\right)^{-1}$ für den Niedrigdosisbereich unter $6 \mathrm{mg} / \mathrm{m}^{3}$ ab [3]. Der sogenannte Knickpunkt von $6 \mathrm{mg} / \mathrm{m}^{3}$ wurde von der ECHA aus dem vom Ausschuss für Gefahrstoffe ermittelten NOAEC von $33 \mathrm{mg} /$ $\mathrm{m}^{3}$ (s. o.) unter Verwendung eines Faktors von 5,3 zur Extrapolation der Exposition vom Arbeitsplatz auf die Exposition der Allgemeinbevölkerung abgeschätzt. Der Extrapolationsfaktor wurde wie folgt errechnet: $20 \mathrm{~m}^{3} \mathrm{~d}^{-1} / 10 \mathrm{~m}^{3} \mathrm{~d}^{-1} \times 7 \mathrm{~d} / 5 \mathrm{~d} \times$ $52 \mathrm{w} / 48 \mathrm{w} \times 70 \mathrm{a} / 40 \mathrm{a}=5,3$. Der Einsatz dieses Extrapolationsfaktors führt zu einem Knickpunkt von $33 \mathrm{mg} / \mathrm{m}^{3}$ : 5,3= (gerundet) $6 \mathrm{mg} / \mathrm{m}^{3}$.

\subsection{Gesundheitliche Bewertung von Trichlorethen in der Innenraumluft}

Nach Ansicht der meisten regulatorischen Gremien sollte Trichlorethen als ein Kanzerogen ohne Wirkungsschwelle angesehen werden. Die Ad-hoc-Arbeitsgruppe Innenraumrichtwerte hält die Begründungen des Ausschusses für Gefahrstoffe, des europäischen Wissenschaftlichen Ausschusses für Expositionsbegrenzungen am Arbeitsplatz und des Risikobewertungsausschusses der Europäischen Chemikalienbehörde zur Annahme eines sublinearen Verlaufs des Krebsrisikos durch chronisch eingeatmetes Trichlorethen für belastbar. Sie hält die Annahme eines linearen Risikomodells der Weltgesundheitsorganisation $[47,48]$ sowie der USUmweltbehörde [49] nicht für angemes- 
sen, sondern folgt der Annahme eines sublinearen Modells der ECHA.

In ihrer Ergänzung zum Basisschema zur gesundheitlichen Bewertung krebserzeugender Stoffe in der Innenraumluft sieht die Ad-hoc-Arbeitsgruppe Innenraumrichtwerte expositionsmindernde Maßnahmen bei einem Krebsrisiko unter $10^{-6}$ nicht als angemessen an [2]. Bei Verwendung des vom Risikobewertungsausschuss der Europäischen Chemikalienbehörde für die Allgemeinbevölkerung nach lebenslanger Exposition im Niedrigdosisbereich (unterhalb von $6 \mathrm{mg} / \mathrm{m}^{3}$ ) abgeschätzten Krebsrisikos durch Trichlorethen von $6,4 \times 10^{-5}\left(\mathrm{mg} / \mathrm{m}^{3}\right)^{-1}$ entspricht ein Krebsrisiko von $10^{-6}$ einer Konzentration von (gerundet) $0,02 \mathrm{mg}$ Trichlorethen $/ \mathrm{m}^{3}$.

Die Konzentration von 0,02 mg Trichlorethen $/ \mathrm{m}^{3}$ liegt über dem 95 . Perzentil einer für Deutschland repräsentativen Studie zum Vorkommen von Trichlorethen in der Innenraumluft von Wohnungen (s. Abschn. 2). Gemäß ihrer Ergänzung zum Basisschema [2] legt die Adhoc-Arbeitsgruppe als risikobezogenen Leitwert für die Innenraumluft eine Konzentration von $0,02 \mathrm{mg}$ Trichlorethen $/ \mathrm{m}^{3}$ fest.

Da es sich bei der krebserzeugenden Wirkung von Trichlorethen um ein Langzeitrisiko handelt, haben einmalige Kurzzeitmessungen von Trichlorethen in der Innenraumluft nur eine eingeschränkte Aussagekraft. Wird im Rahmen einer Kurzzeitmessung der risikobezogene Leitwert überschritten, sind für eine Bewertung des Risikos und zur Ableitung angemessener Maßnahmen weitergehende Untersuchungen notwendig.

Es wird empfohlen, Trichlorethen in der Innenraumluft über mindestens eine Woche z. B. mit einem Passivsammler zu erfassen. Für Innenraumarbeitsplätze und Innenräume mit eindeutig festgelegten Nutzungszeiten wird die Probenahme außerhalb dieser Zeiten, z. B. in den Nächten und am Wochenende, unterbrochen. Sollte bei der Langzeitprobenahme der Innenraumluft der risikobezogene Leitwert überschritten werden, wird das Ergebnis durch eine Wiederholungsmessung unter üblichen Nutzungsbedingungen überprüft. Ergibt auch die Wiederholungsmessung eine Überschreitung des Leitwertes, sollten expositionsmindernde Maßnahmen eingeleitet werden, deren Wirksamkeit zu überprüfen ist.

\section{Anmerkungen}

Der Text dieser Veröffentlichung wurde von Dr. Helmut Sagunski, Dr. Ludwig Müller und Herrn Herbert Grams mit Beiträgen von Herrn Thomas Lahrz erarbeitet und von der Ad-hoc-Arbeitsgruppe im Februar 2015 verabschiedet. Die Literaturrecherche wurde im Juli 2014 abgeschlossen.

\section{Literatur}

1. Verordnung (EG) Nr. 1272/2008 des Europäischen Parlaments und des Rates vom 16. Dezember 2008 über die Einstufung, Kennzeichnung und Verpackung von Stoffen und Gemischen, zur Änderung und Aufhebung der Richtlinien 67/548/EWG und 1999/45/EG und zur Änderung der Verordnung (EG) Nr. 1907/2006. Amtsbl EU L 353/1 vom 31.12.2008

2. Ad-hoc-Arbeitsgruppe Innenraumrichtwerte der IRK/AOLG (2015) Gesundheitliche Bewertung krebserzeugender Verunreinigungen der Innenraumluft: erste Ergänzung zum Basisschema. Bundesgesundheitsbl 58, in Vorbereitung

3. ECHA (2014) Application for authorisation: establishing a reference dose response relationship for carcinogenicity of trichloroethylene. RAC/28/2014/07 rev 2 final. European Chemicals Agency. Committee for Risk Assessment. http:// echa.europa.eu/documents/10162/13641/carcinogenicity dose response tce en.pdf

4. IARC (2014) Trichloroethylene, tetrachloroethylene, and some chlorinated agents. IARC Monographs on the evaluation of carcinogenic risks to humans. Vol 106, 35-217. International Agency for Research on Cancer. World Health Organization, Lyon.

5. EU-SCOEL (2009) Recommendation from the Scientific Committee on occupational exposure limits for trichloroethylene. SCOEL/SUM/142. April 2009

6. 2 BImSchV (1990) Zweite Verordnung zur Durchführung des Bundes-Immissionsschutzgesetzes (Verordnung zur Emissionsbegrenzung von leichtflüchtigen halogenierten organischen Verbindungen - 2. BImSchV) vom 10. Dezember 1990 (BGBI. I S. 2694), zuletzt geändert durch Artikel 1 der Verordnung vom 2. Mai 2013 (BGBI. I S. 1021, 3754)

7. Umweltbundesamt (2008) Vergleichswerte für flüchtige organische Verbindungen (VOC und Aldehyde) in der Innenraumluft von Haushalten in Deutschland. Bundesgesundheitsbl 51: 109-112

8. Hofmann H, Plieninger P (2008)) Bereitstellung einer Datenbank zum Vorkommen von flüchtigen organischen Verbindungen in der Raumluft. Forschungsbericht 20561 243. Arbeitsgemeinschaft ökologischer Forschungsinstitute (AGÖF) e. V. im Auftrag des Umweltbundesamts. WaBoLu-Hefte 05/08. Umweltbundesamt, Dessau-Roßlau

9. Lock EA, Reed CJ (2006) Trichloroethylene: mechanisms of renal toxicity and renal cancer and relevance to risk assessment. Toxicol Sci 91:313-331
10. Rusyn I, Chiu WA, Lash LH et al (2014) Trichloroethylene: mechanistic, epidemiologic and other supporting evidence of carcinogenic hazard. Pharmacol Therap 141:55-68

11. Kezic S, Monster AC, Krüse J, Verbeck MM (2000) Skin absorption of some vaporous solvents in volunteers. Int Arch Occup Environ Health 73:415422

12. Birner $G$, Vamvakas S, Dekant W, Henschler D (1993) Nephrotoxic and genotoxic N-Acetyl-S-dichlorvinyl-L-cystein is a urinary metabolite after occupational 1,1,2-trichloroethene exposure in humans: implication for the risk of trichloroethene exposure. Environ Health Perspect 99:281-284

13. Bernauer U, Birner G, Dekant W, Henschler D (1996) Biotransformation of trichloroethene: dosedependent excretion of 2,2,2-trichloro-metabolites and mercapturic acids in rats and humans after inhalation. Arch Toxicol 70:338-346

14. Bloemen LJ, Monster AC, Kezic S et al (2001) Study on the cytochrome P-450- and glutathione-dependent biotransformation of trichloroethylene in humans. Int Arch Occup Environ Health 74:102108

15. Lash LH, Fisher JW, Lipscomb JC, Parker JC (2000) Metabolism of trichloroethylene. Environ Health Perspect 108 Suppl 2:177-200

16. Commandeur JNM, Vermeulen NPE (1990) Identification of N-Acetyl(2,2-dichlorvinyl)- and N-Acetyl(1,2-dichlorvinyl)-L-cystein as two regioisomeric mercapturic acids of trichloroethylene in the rat. Chem Res Toxicol 3:212-218

17. Lash LH, Putt DA, Brashear WT et al (1999) Identification of S-(1,2-dichlorovinyl)glutathione in the blood of human volunteers exposed to trichloroethylene. J Toxicol Environ Health A 56:1-21

18. Kim S, Kim D, Pollack GM et al (2009) Pharmacokinetik analysis of trichloroethylene metabolism in male B6C3F1 mice: formation and disposition of trichloroacetic acid, dichloroacetic acid, S-(1,2dichlorovinyl)glutathione and S-(1,2-dichlorovinyl)-L-cystein. Toxicol Appl Pharmacol 238:90-99

19. Green T, Dow J, Ellis MK et al. (1997) The role of glutathione conjugation in the development of kidney tumours in rats exposed to trichloroethylene. Chem Biol Interact 105:99-107

20. Fisher JW, Mahle D, Abbas R (1998) A human physiologically-based pharmacokinetic model for trichloroethylene and its metabolites, trichloroacetic acid and free trichloroethanol. Toxicol Appl Pharmacol 152:339-359

21. Harth V, Brüning T, Bolt HM (2005) Renal carcinogenicity of trichloroethylene: update, mode of action, and fundamentals for occupational standard setting. Rev Environ Health 20:103-118

22. Müller G, Spassowski M, Henschler D (1975) Metabolim of trichloroethylene in man. III. Interaction of trichloroethylene and ethanol. Arch Toxicol 33:173-189

23. Sellers EM, Lang M, Koch-Weser J et al (1972) Interaction of chloral hydrate and ethanol in man. I. Metabolism. Clin Pharmacol Ther 13:37-49

24. Nakajima T, Wang RS, Elovaara E et al. (1992) A comparative study on the contribution of cytochrome P450 isozymes to metabolism of benzene, toluene and trichloroethylene in rat liver. Biochem Pharmacol 43:251-257

25. Chiu WA, Jinot J, Scott CS et al. (2012) Human health effects of trichlororethylene: key findings and scientific issues. Environ Health Perspect 121:303-311

26. Landrigan PJ, Kominsky JR (1987) Common-source community and industrial exposure to trichloroethylene. Arch Environ Health 42:327-332 
27. Murata K, Inoue O, Akutsu M, Iwata T (2010) Neuromotor effects of short-term and long-term exposures to trichloroethylene in workers. Am J Ind Med 53:915-921

28. Green T, Dow J, Ong CN et al (2004) Biological monitoring of kidney function among workers occupationally exposed to trichlororethylene. Occup Environ Med 61:312-317

29. Seldén A, Hultberg B, Ulander A, Ahlborg G Jr (1993) Trichlororethylene exposure in vapour degreasing and the urinary excretion of $\mathrm{N}$-acetyl-beta-D-glucosaminidase. Arch Toxicol 67:224-226

30. Vermeulen R, Zhang L, Spierenburg A et al (2012) Elevated urinary levels of kidney injury molecule-1 among Chinese factory workers exposed to trichlororethylene. Carcinogenesis 33:1538-1541

31. Scott CS, Jinot J (2011) Trichloroethylene an cancer: systematic and quantitative review of epidemiologic evidence for identifying hazards. Int J Environ Res Public Health 8:4238-4272

32. Karami S, Lan Q, Rothman N et al (2012) Occupational trichloroethylene exposure and kidney cancer risk: a meta-analysis. Occup Environ Med 69:858-867

33. Henschler D, Vamvakas S, Lammert M et al (1995) Increased incidence of renal cell tumors in a cohort of cardboard workers exposed to trichloroethylene. Arch Toxicol 69:291-299

34. Raaschou-Nielsen O, Hansen J, McLaughlin JK et al (2003) Cancer risk among workers of Danish companies using trichloroethylene: a cohort study. Am J Epidemiol 158:1182-1192

35. Moore LE, Bofetta P, Karami S et al (2010) Occupational trichloroethylene exposure and renal carcinoma risk: evidence of genetic susceptibility by reductive metabolism gen variants. Cancer Res 70(16):6527-6536

36. Purdue MP, Bakke B, Stewart P et al (2011) A casecontrol study of occupational exposure to trichloroethylene and non-Hodgkin lymphoma. Environ Health Perspect 119(2):232-238

37. Guha N, Loomis D, Grosse Y et al (2012) Carcinogenicity of trichloroethylene, tetrachloroethylene, some other chlorinated solvents, and their metabolites. Lancet Oncol 13:1192-1193

38. Maltoni C, Lefemine G, Cotti G, Perino G (1988) Long-term carcinogenicity bioassays on trichloroethylene administered by inhalation to SpragueDawley rats and Swiss and B6C3F1 mice. Ann NY Acad Sci 534:316-342

39. Henschler D, Romen W, Elsässer HM et al (1980) Carcinogenicity study of trichloroethylene by long term inhalation in three animal species. Arch Toxicol 43:237-248

40. US-NTP (1988) Toxicology and carcinogenesis studies of trichloroethylene (CAS No. 79-01-6) in four strains of rats (ACl, August, Marshall, OsborneMendel) (gavage studies). Natl Toxicol Program Tech Rep Ser 273:1-299

41. US-NTP (1990) Carcinogenesis studies of trichloroethylene (without epichlorohydrin) (CAS No. 7901-6) in F344/N rats and B6C3F1 mice (gavage studies). Natl Toxicol Program Tech Rep Ser 243:1-174

42. US-NTP (2011) Trichlororethylene. Rep Carcinog $12: 420-423$

43. Vamvakas S, Dekant W, Henschler D (1989) Assessment of unscheduled DNA synthesis in a cultured line of renal epithelial cells exposed to cysteine $S$ conjugates of haloalkenes and haloalkanes. Mutat Res 222:329-335

44. Abe K (1988) Determination of olfactory threshold values of organic solvents for the control of the working environment. J Working Environ 9:57-62
45. Nagata Y (2003) Measurement of odor threshold by triangle odor bag method. Japan Ministry of the Environment 118-127. http://www.env.go.jp/ en/air/odor/measure/02_3_2.pdf

46. AGS (2008) Begründung zu Expositions-Risiko-Beziehung für Trichlorethen in BekGS 910. Ausschuss für Gefahrstoffe. http://www.baua.de/de/Themenvon-A-Z/Gefahrstoffe/TRGS/Begruendungen-910. html;jsessionid=21B49E5B1BC6BC83E21E4577845 16DEF.1_cid343. Zugegriffen: 21.01.2015

47. WHO (2000) Air quality guidelines for Europe, 2nd Ed. Trichloroethylene. World Health Organization, European Series No. 91, Copenhagen

48. WHO (2010) WHO guidelines for indoor air quality: selected pollutants. Trichloroethylene. World Health Organization, Regional Office for Europe, Copenhagen: 377-414

49. US-EPA (2011) Trichlororethylene. Toxicological review of TRICHLOROETHYLENE (CAS No. 79-01-6) In Support of Summary Information on the Integrated Risk Information System (IRIS). September 2011. EPA/635/R-09/011F

50. Charbotel B, Fevotte J, Hours M et al (2006) Casecontrol study on renal cell cancer and occupational exposure to trichloroethylene. Part II: Epidemiological aspects. Ann Occup Hyg 50:777-787

51. Vamvakas S, Brüning T, Thomasson B et al (1998) Renal cell cancer correlated with occupational exposure to trichloroethylene. J Cancer Res Clin Oncol 124:374-382

52. Brüning T, Pesch B, Wiesenhütter $B$ et al (2003) Renal cell cancer risk and occupational exposure to trichloroethylene: results of a consecutive casecontrol study in Arnsberg, Germany. Am J Ind Med 43:274-285 